Supplementary Information

\title{
Biomimetic Z-scheme Photocatalyst with a Tandem Solid-state Electron Flow Catalyzing $\mathrm{H}_{2}$ Evolution
}

Weilai $\mathrm{Yu}^{\mathrm{a}, \mathrm{b}^{*}}$, Shuai Zhang ${ }^{\mathrm{a}}$, Junxiang Chen ${ }^{\mathrm{a}}$, Pengfei $\mathrm{Xia}^{\mathrm{c}}$, Matthias H. Richter ${ }^{\mathrm{b}}$, Linfeng Chen ${ }^{\mathrm{a}}$, Wei X $\mathrm{u}^{\mathrm{a}}$, Jingpeng Jin ${ }^{\mathrm{a}}$, Shengli Chen ${ }^{\mathrm{a}}$, Tianyou Peng ${ }^{{ }^{*}}$

${ }^{a}$ College of Chemistry and Molecular Sciences, Wuhan University, Wuhan, 430072,

China

${ }^{b}$ Division of Chemistry and Chemical Engineering, California Institute of Technology,

Pasadena, CA, 91125, USA

${ }^{c}$ State Key Laboratory of Advanced Technology for Material Synthesis and

Processing, Wuhan University of Technology, Wuhan, 430070, China

Email: yuweilai93@whu.edu.cn; typeng@whu.edu.cn 


\section{Experimental}

\subsection{Materials syntheses}

All reagents were of analytical grade and used without further purification.

Preparation of CdS NWs: CdS NWs were synthesized thorough a ethylenediamineassisted solvothermal method. In a typical synthesis, $3.00 \mathrm{~g}$ of cadmium nitrate tetrahydrate $\left(\mathrm{Cd}\left(\mathrm{NO}_{3}\right)_{2} .4 \mathrm{H}_{2} \mathrm{O}\right)$ was dissolved in $65 \mathrm{~mL}$ of ethylenediamine under vigorous stirring in a $100 \mathrm{~mL}$ Teflon-lined autoclave. Then, $2.20 \mathrm{~g}$ of thiourea was added into the above solution and continuous stirring was maintained for 10 min until it was fully dissolved. The autoclave was sealed tightly and put into oven at $200{ }^{\circ} \mathrm{C}$ for $24 \mathrm{~h}$. Afterwards, the obtained yellow precipitates were centrifuged, rinsed with ethanol and distilled water three times and dried in oven at $70{ }^{\circ} \mathrm{C}$ overnight.

Preparation of CdS-CBP composites: the CdS-CBP composites were fabricated via a one-step procedure in solution phase. In a beaker, $0.291 \mathrm{~g}$ of cobalt nitrate hexahydrate $\left(\mathrm{Co}\left(\mathrm{NO}_{3}\right)_{2} \cdot 6 \mathrm{H}_{2} \mathrm{O}\right)$ was dissolved in $30 \mathrm{~mL}$ of methanol to form a light red solution and $0.20 \mathrm{~g}$ of the above CdS NWs was then added into the solution. Meanwhile, in another beaker, $0.944 \mathrm{~g}$ of benzimidazole was dissolved in $30 \mathrm{~mL}$ of methanol to prepare a colorless transparent solution. After stirring for $1 \mathrm{~h}$, the benzimidazole/methanol solution was rapidly poured into the suspension containing CdS NWs and $\mathrm{Co}^{2+}$ ions. Then the color of the suspension was observed to change immediately. The suspension was stirred for another specific period of time before the resulting solids was centrifuged and rinsed with ethanol three times. Different samples were named as CdS-CBP- $x \mathrm{~h}$, where $x$ represented the stirring time (number of hours) for the syntheses of composites. For the purpose of comparison, pure CBPs were also 
obtained in the absence of CdS NWs under the same conditions, which were named as CBP-xh.

Method of loading 1 wt.\% Pt co-catalyst onto CdS NWs: 0.2 g of CdS NWs was put in $50 \mathrm{~mL}$ aqueous solution containing $133.6 \mu \mathrm{L}$ chloroplatinic $\left(\mathrm{H}_{2} \mathrm{PtCl}_{6}\right)$ solution $(0.077 \mathrm{M})$. The suspension was first maintained stirring in dark for $30 \mathrm{~min}$. Then $0.1 \mathrm{~g}$ of $\mathrm{NaBH}_{4}$ was added into the suspension for reducing platinum and continuous stirring was kept for another 3 hours. After the reaction was complete, the containing solids were centrifuged, rinsed with water and ethanol three times and dried in oven at $70{ }^{\circ} \mathrm{C}$ overnight.

\subsection{Characterization}

X-ray diffraction (XRD) patterns were obtained on Miniflex600 X-ray diffractometer (Rigaku, Japan) with $\mathrm{Cu} \mathrm{K} \alpha$ radiation at the accelerating voltage of $40 \mathrm{kV}$. A scan rate of $4^{\circ} \mathrm{min}^{-1}$ was applied to record the powder patterns for $2 \theta$ in the range of $5-60^{\circ}$. UV-vis diffusive reflectance spectra (DRS) were obtained on a MPC-3100 spectrometer equipped with a integrating sphere (Shimadzu, Japan) by using diepressed disk samples. Specpure $\mathrm{BaSO}_{4}$ was used as the reflectance standard sample for measurements. Thermogravimetric analysis (TGA) of the samples were performed on DTG-60H analyzer (Shimadzu, Japan) from ambient temperature to $900{ }^{\circ} \mathrm{C}$ at a heating rate of $10{ }^{\circ} \mathrm{C} \min ^{-1}$. X-ray photoelectron spectroscopy (XPS) measurements were performed on a ESCALAB $250 \mathrm{Xi}$ electron spectrometer (Thermo Fisher, USA) equipped with $\mathrm{Al} \mathrm{K \alpha}$ radiation source operated at $200 \mathrm{~W}$. All the binding energies were referenced to the $\mathrm{C} 1 \mathrm{~s}$ peak at $284.8 \mathrm{eV}$ of the surface adventitious carbon. The ultraviolet photoelectron spectroscopy (UPS) was performed on a Kratos Axis Ultra 
system with a base pressure of $1 \times 10^{-9}$ Torr in the analysis chamber. Field-emission scanning electron microscopy (FE-SEM) images, EDX mapping and line scan profiles were obtained on JSM-7500F (JEOL, Japan) scanning electron microscope. Transmission electron microscopy (TEM) images and high-resolution transmission electron microscopy (HR-TEM) analyses were performed on JEM-2100HR (JEOL, Japan) at an accelerating voltage of $200 \mathrm{kV}$. Photoluminescence (PL) emission spectra were measured at room temperature on F-7000 fluorescence spectrometer (Hitachi, Japan) with excitation wavelength of $375 \mathrm{~nm}$. Nitrogen adsorption-desorption isotherms were measured on an ASAP 2020 nitrogen adsorption apparatus (Micromeritics, USA). All of the samples were degassed at $180{ }^{\circ} \mathrm{C}$ before measurements. The BET surface area was determined by a multipoint BET method using the adsorption date in the relative pressure $\left(\mathrm{P} / \mathrm{P}_{0}\right)$ range of $0.05-0.25$. The pore size distributions were determined using the desorption date by the Barrett-JoynerHalenda (BJH) method. Electron paramagnetic resonance (EPR) spectra are performed on an ESR spectrometer (MEX-nano, Bruker) with a modulation frequency of $100 \mathrm{kHz}$ and a microwave power of $15 \mathrm{~mW}$. The hydroxyl radicals $(\cdot \mathrm{OH})$ and superoxide anion radicals $\left(\cdot \mathrm{O}_{2}^{-}\right)$are trapped by the 5,5-dimethyl-1-pyrroline $\mathrm{N}$-oxide (DMPO), producing the ESR signals of their adducts. The mixed solution of DMPO/ $\mathrm{H}_{2} \mathrm{O}$ and DMPO/ $\mathrm{CH}_{3} \mathrm{OH}$ was prepared by the traditional method. Briefly, $10 \mathrm{mg}$ asfabricated samples and $40 \mu \mathrm{L}$ DMPO were dissolved in $1 \mathrm{~mL}$ deionized water under the stirring condition. Then the mixture was irradiated under a $300 \mathrm{~W}$ xenon lamp for 120s. After that, $0.1 \mathrm{~mL}$ mixture solution (containing DMPO-•OH) was quickly detected by the ESR spectrometer. The detection method of DMPO- $\cdot \mathrm{O}_{2}{ }^{-}$was identical to the DMPO-•OH, except that the deionized water was replaced by the methanol $\left(\mathrm{CH}_{3} \mathrm{OH}\right)$. 


\subsection{Photocatalytic $\mathrm{H}_{2}$ production activity test}

Photocatalytic $\mathrm{H}_{2}$ production tests were performed in an outer irradiation-type photoreactor (Pyrex glass) connected to a closed gas-circulation system. A $300 \mathrm{~W}$ Xe lamp (PLS-SXE300, Beijing Trusttech Co. Ltd., China) was used as light source, which was collimated and focalized into $5 \mathrm{~cm}^{2}$ parallel faculae. The illuminated spectrum of incident light of the lamp is shown in Figure S11. Typically, the photoreaction is performed in $10 \mathrm{~mL}$ of aqueous suspension containing $5 \mathrm{mg}$ of photocatalyst and 10 vol\% of triethanolamine (TEOA) as sacrificial reagent. Prior to irradiation, the suspension of photocatalyst was dispersed in an ultrasonic bath for $5 \mathrm{~min}$ and then the reactor was irradiated from top after the air had been thoroughly removed. The produced $\mathrm{H}_{2}$ was analyzed with a gas chromatograph (GC, SP6890, TCD detector, 5 $\AA$ molecular sieve columns and Ar carrier). During photocatalytic activity stability test, due to the volume loss of suspension in reactor, the suspension was refreshed with TEOA solution after each cycle to restore its volume to $10 \mathrm{~mL}$.

\subsection{Photoelectrochemical measurements}

Measurements of photocurrent response, electrochemical impedance spectra (EIS) and Mott-Schottky plots were performed on a CHI 660D electrochemical workstation (Chenhua Instrument, Shanghai, China) in a conventional three-electrode configuration, including a working electrode, counter electrode and reference electrode. The working electrodes were prepared with as-prepared photocatalysts with an active area of $c a .1 .1 \mathrm{~cm}^{2}$. A Pt wire and $\mathrm{Ag} / \mathrm{AgCl}$ electrode (immersed in saturated $\mathrm{KCl}$ solution) were used as the counter electrode and reference electrode, respectively. 
Low-power LED with wavelength of $420 \mathrm{~nm}$ (3 W, Shenzhen LAMPLIC Science Co. Ltd China) were used as the light source with a focused intensity of $c a .80 .0 \mathrm{~mW} . \mathrm{cm}^{-2}$. 0.5 $\mathrm{M} \mathrm{Na}_{2} \mathrm{SO}_{4}$ aqueous solution was used as the electrolyte in photoelectrochemical measurement. Typically, the working electrodes were prepared as follows: $10 \mathrm{mg}$ of sample was ground with ethanol to form a slurry. Then the slurry was coated onto a pre-cleaned $2 \mathrm{~cm}$ x $1.2 \mathrm{~cm}$ F-doped $\mathrm{SnO}_{2}$-coated glass (FTO glass) electrode via a doctor blade technique. Last, the prepared electrodes were dried naturally overnight before measurements.

\subsection{Computational Details}

Geometry optimization and total energy calculations were performed within DFT framework as implemented with $\mathrm{DMol}^{3}$ code. The spin is unrestricted during the calculation. The PBE exchange-correlation functional within the generalized gradient approximation (GGA) was introduced. The all electron method was adopted. A double numerical basis set was used together with polarization function (DNP). A convergence criterion of $1 \times 10^{-6} \mathrm{Ha}(1 \mathrm{Ha}=27.21 \mathrm{eV})$ on the total energy has been performed in the self-consistent-field (SCF) procedures. The Broyden-FletcherGoldfarb-Shanno (BFGS) algorithm was used in performing the geometry optimizations. The convergence tolerances of energy, displacement and forces were $1 \times 10^{-5} \mathrm{Ha}, 0.005 \AA$ and $0.002 \mathrm{Ha} / \AA$, respectively in geometry optimization. A smearing of $0.005 \mathrm{Ha}$ to the orbital occupation is applied to achieve accurate electronic convergence. 


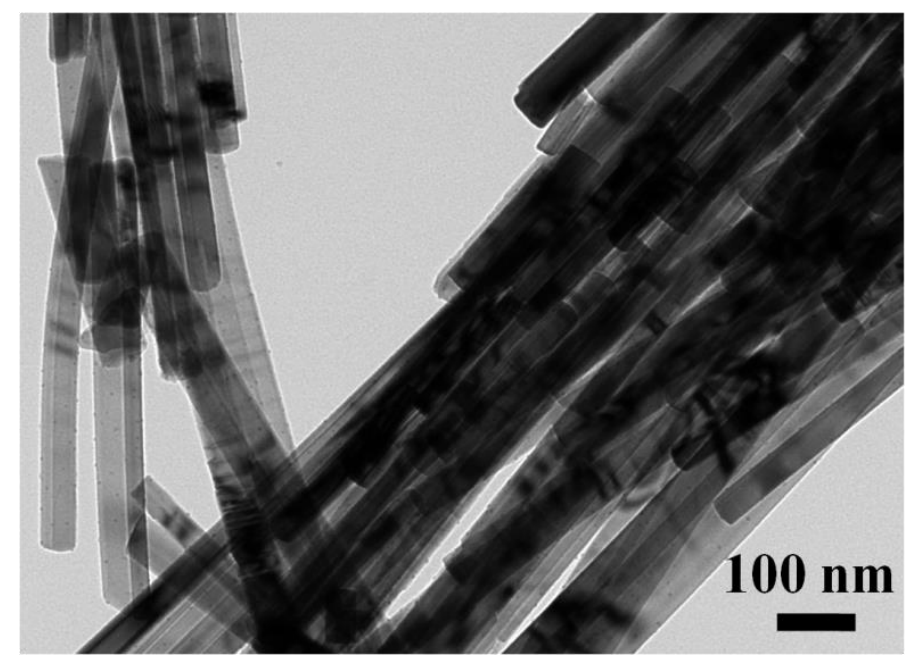

Figure S1. TEM image of CdS NWs 

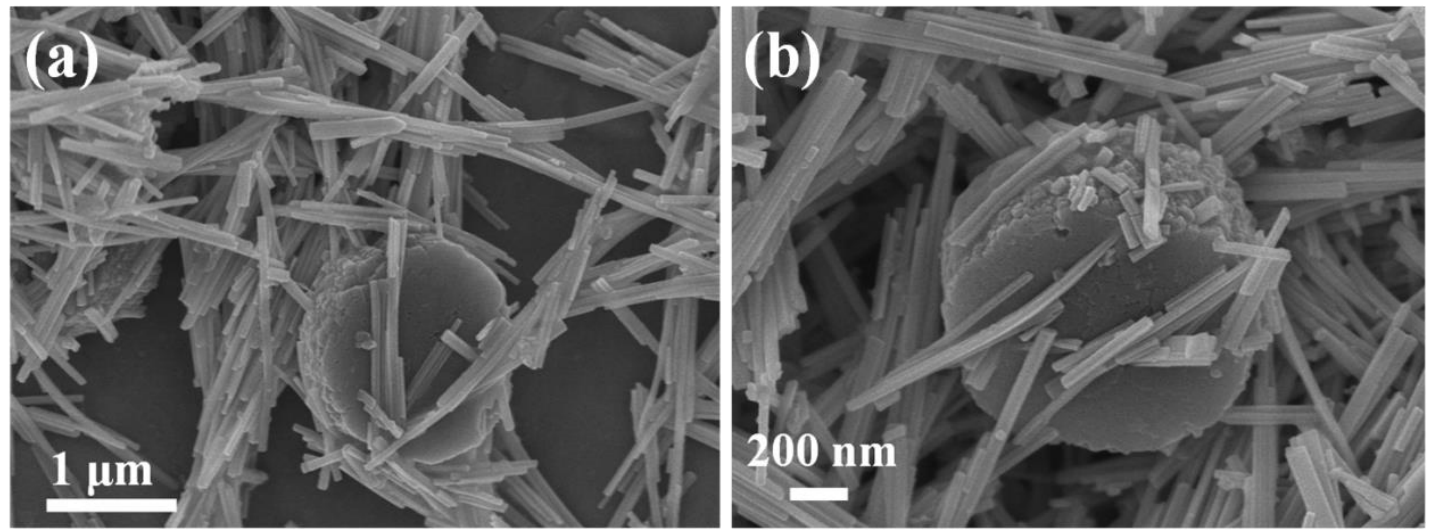

Figure S2. (a-b) FE-SEM images of the CdS-CBP-1h composite, showing the rough and jaggy edges of CBP microstructures 


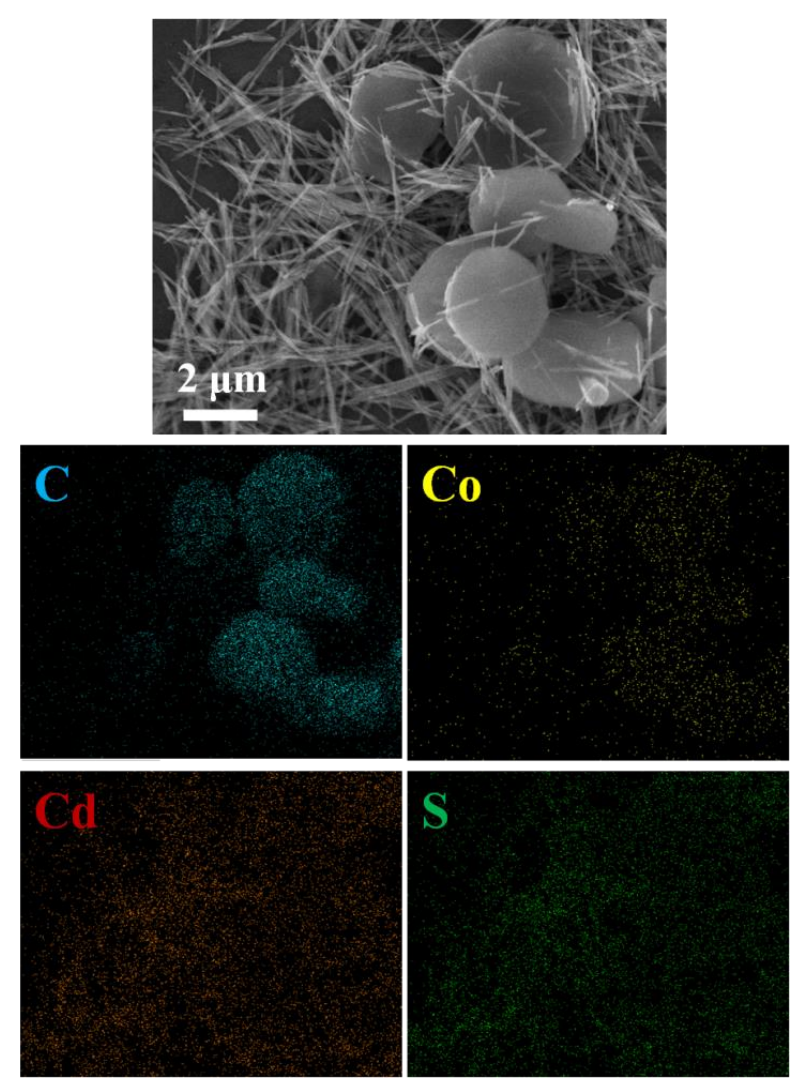

Figure S3. EDS mapping profile of the CdS-CBP-6h composite
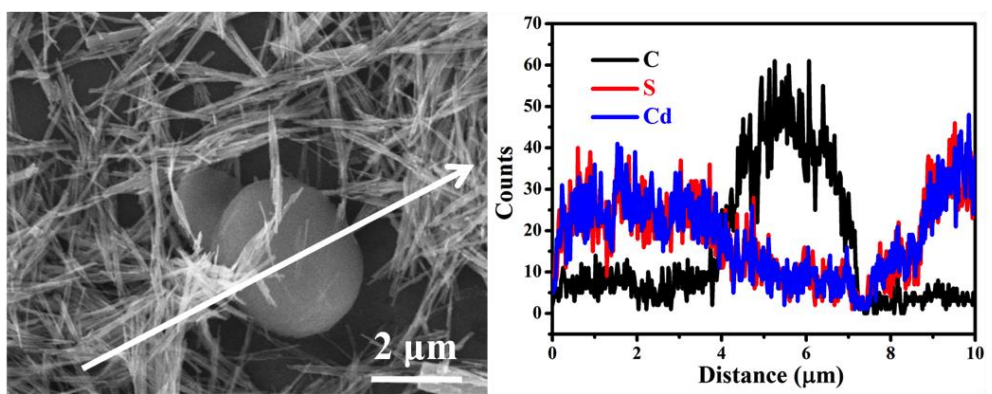

Figure S4. Line scan profile of the CdS-CBP-6h composite 


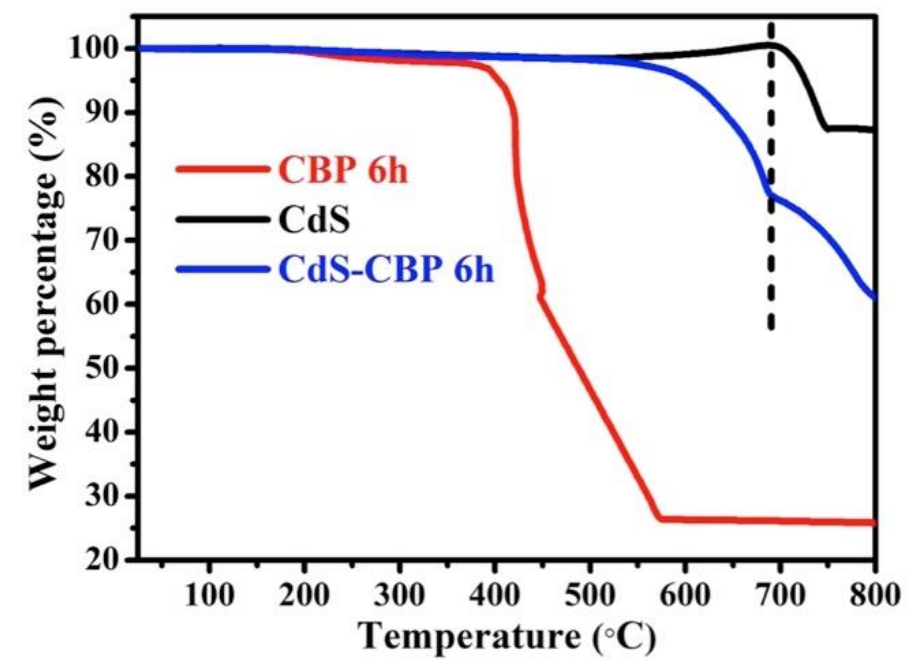

Figure S5. TGA curves of pristine CdS (black), pure CBP 6h (red) and CdS-CBP $6 \mathrm{~h}$ composite (blue)

As shown in Figure S5, pure CBP $6 \mathrm{~h}$ is very stable at temperature below $400{ }^{\circ} \mathrm{C}$ with only negligible weight loss. This showed that the amount of residual methanol in the CBP could be ignored, indicating the formation of CBP coordination polymer did not require the support of external solvent molecule. Then from $400{ }^{\circ} \mathrm{C}$ to $570{ }^{\circ} \mathrm{C}$, its weight percentage underwent drastic decrease from nearly $100 \%$ to $25 \%$, representing the thermal decomposition of bIm and the oxidation of cobalt (II) into $\mathrm{Co}_{3} \mathrm{O}_{4}$. Pure $\mathrm{CdS}$ underwent a decrease of weight percentage to $87 \%$ at temperature above $700{ }^{\circ} \mathrm{C}$, which is consistent with the transformation of $\mathrm{CdS}$ into $\mathrm{CdO}$.

In this regard, the different temperature ranges for the weight loss of pure $\mathrm{CdS}$ and pure CBP can be used to estimate the composition of CdS-CBP 6h composite. The starting temperature for the weight loss of CdS-CBP $6 \mathrm{~h}$ increased to $550{ }^{\circ} \mathrm{C}$, showing an increase of thermal stability. As denoted by dashed line, the change of TGA curve slope at $c a .700{ }^{\circ} \mathrm{C}$ indicated the beginning of weight loss of CdS. The weight loss at the above critical point was $76 \%$, which should be mainly attributed to 
the thermal decomposition of CBP. Thus, the weight content of CBP within the CdSCBP 6h composite was calculated to be $32 \%$. 


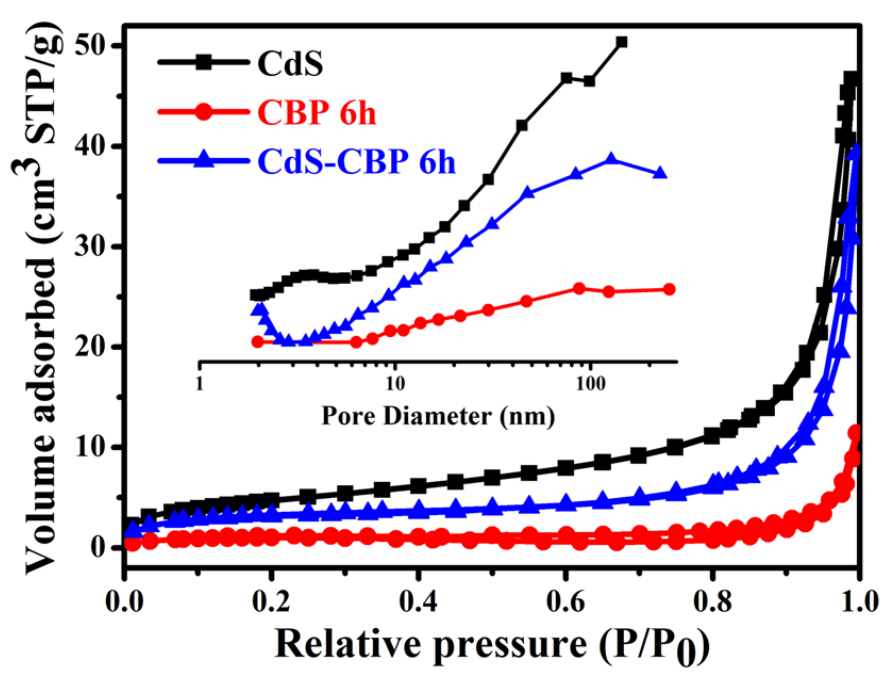

Figure S6. Nitrogen adsorption-desorption isotherms and pore size distributions (inset) of pristine CdS (black), pure CBP 6h (red) and CdS-CBP 6h (blue)

Table S1. Specific surface area, pore volume and pore diameter of pristine CdS, pure CBP 6h and CdS-CBP 6h

\begin{tabular}{cccc}
\hline Sample & $\mathbf{S}_{\text {BET }} / \mathbf{m}^{\mathbf{2}} \mathbf{g}^{-\mathbf{1}}$ & $\mathbf{V}_{\text {pore }} / \mathbf{c m}^{\mathbf{3}} \mathbf{g}^{-\mathbf{1}}$ & $\mathbf{d}_{\text {pore }} / \mathbf{n m}$ \\
\hline CdS & 17 & 0.046 & 10.7 \\
CBP 6h & 4 & 0.008 & 9.2 \\
CdS-CBP 6h & 12 & 0.03 & 10.2
\end{tabular}




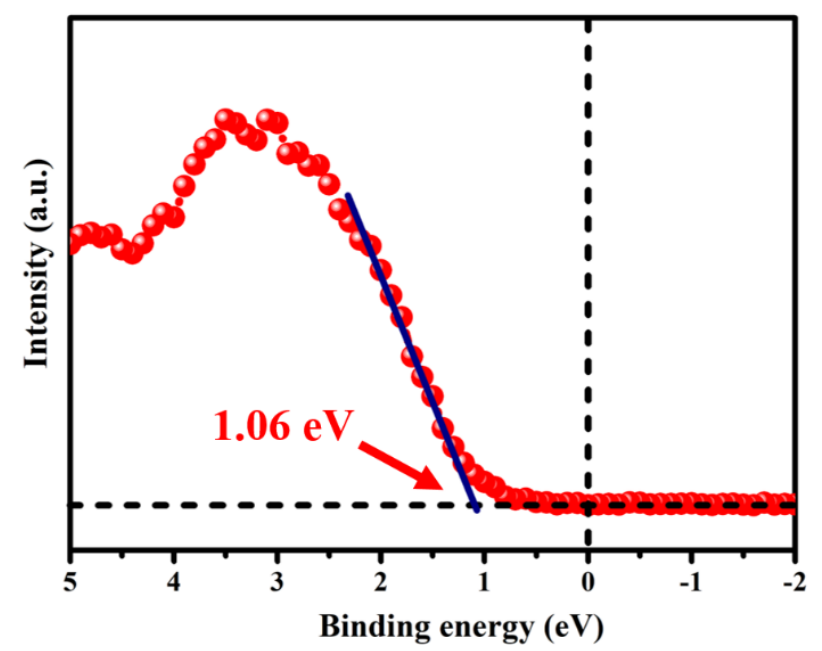

Figure S7. XPS valence band spectrum for CBP 6h, showing the VB is $1.06 \mathrm{eV}$ below its Fermi level

Table S2. Performance comparison with recent efforts of CdS-based photocatalysts for $\mathrm{H}_{2}$ production

\begin{tabular}{|c|c|c|c|c|c|}
\hline Photocatalyst & $\begin{array}{l}\text { Sacrificial } \\
\text { agent }\end{array}$ & $\begin{array}{c}\text { Co- } \\
\text { catalyst }\end{array}$ & $\begin{array}{c}\text { Champion } \mathrm{H}_{2} \\
\text { production } \\
\text { rate/umol/h/g }\end{array}$ & $\begin{array}{c}\text { Improvement } \\
\text { factor over } \\
\text { CdS } \\
\end{array}$ & Ref \\
\hline CdS-CBP & TEOA & none & 97600 & 10.6 & $\begin{array}{l}\text { this } \\
\text { work }\end{array}$ \\
\hline $\mathrm{CdS}-\mathrm{WO}_{3}$ & $\mathrm{Na}_{2} \mathrm{~S} / \mathrm{Na}_{2} \mathrm{SO}_{3}$ & $\mathrm{Pt}$ & 15522 & 2 & 1 \\
\hline CdS-g- $\mathrm{C}_{3} \mathrm{~N}_{4}$ & $\mathrm{Na}_{2} \mathrm{~S} / \mathrm{Na}_{2} \mathrm{SO}_{3}$ & $\mathrm{Pt}$ & 5690 & & 2 \\
\hline $\mathrm{CdS}-\mathrm{Bi}_{2} \mathrm{MoO}_{6}$ & $\mathrm{Na}_{2} \mathrm{~S} / \mathrm{Na}_{2} \mathrm{SO}_{3}$ & $\mathrm{Pt}$ & 7370 & 5.5 & 3 \\
\hline CdS-Au-g- $\mathrm{C}_{3} \mathrm{~N}_{4}$ & methanol & $\mathrm{Pt}$ & 19.02 & & 4 \\
\hline $\mathrm{CdS}-\mathrm{TiO}_{2}$ & methanol & & 1028 & & 5 \\
\hline $\mathrm{CdS}-\mathrm{SiC}$ & $\mathrm{Na}_{2} \mathrm{~S} / \mathrm{Na}_{2} \mathrm{SO}_{3}$ & & 259 & & 6 \\
\hline $\mathrm{CdS}-\mathrm{Ti}_{3} \mathrm{C}_{2}$ & lactic acid & & 14342 & & 7 \\
\hline CdS-Phosphorene & lactic acid & & 11192 & 11.6 & 8 \\
\hline $\mathrm{CdS}_{-\mathrm{BiVO}_{4}}$ & $\mathrm{Na}_{2} \mathrm{~S} / \mathrm{Na}_{2} \mathrm{SO}_{3}$ & $\mathrm{Pt}$ & 57000 & 5.2 & 9 \\
\hline $\mathrm{CdS}-\mathrm{MoS}_{2}$ & $\mathrm{Na}_{2} \mathrm{~S} / \mathrm{Na}_{2} \mathrm{SO}_{3}$ & $\mathrm{MoS}_{2}$ & 1750 & 2.03 & 10 \\
\hline $\mathrm{CdS}-\mathrm{BiVO}_{4}$ & $\mathrm{Na}_{2} \mathrm{SO}_{3}$ & $\mathrm{Pt}$ & 23060 & 2.03 & 11 \\
\hline CdS-CoPi & lactic acid & & 13300 & 2.6 & 12 \\
\hline CdS-red P & $\mathrm{Na}_{2} \mathrm{~S} / \mathrm{Na}_{2} \mathrm{SO}_{3}$ & $\mathrm{Pt}$ & 923 & 2.53 & 13 \\
\hline $\mathrm{CdS} / \mathrm{WO}_{3}$ & lactic acid & $\mathrm{Pt}$ & 2900 & 5 & 14 \\
\hline
\end{tabular}




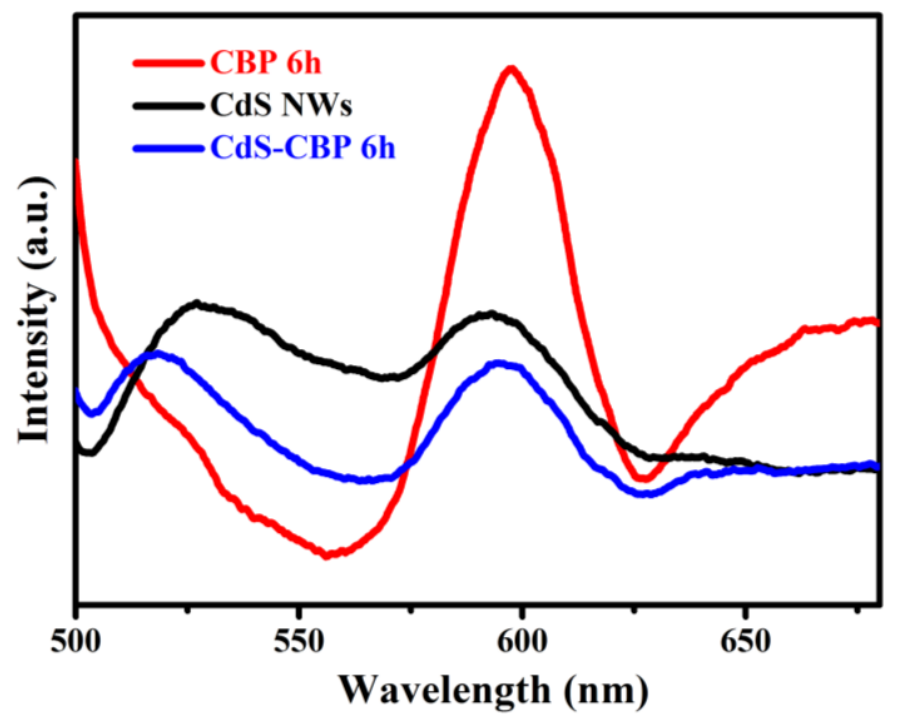

Figure S8. Comparison of PL spectra of pristine CdS NWs (black), pure CBP 6h (red) and CdS-CBP-6h composite (black)
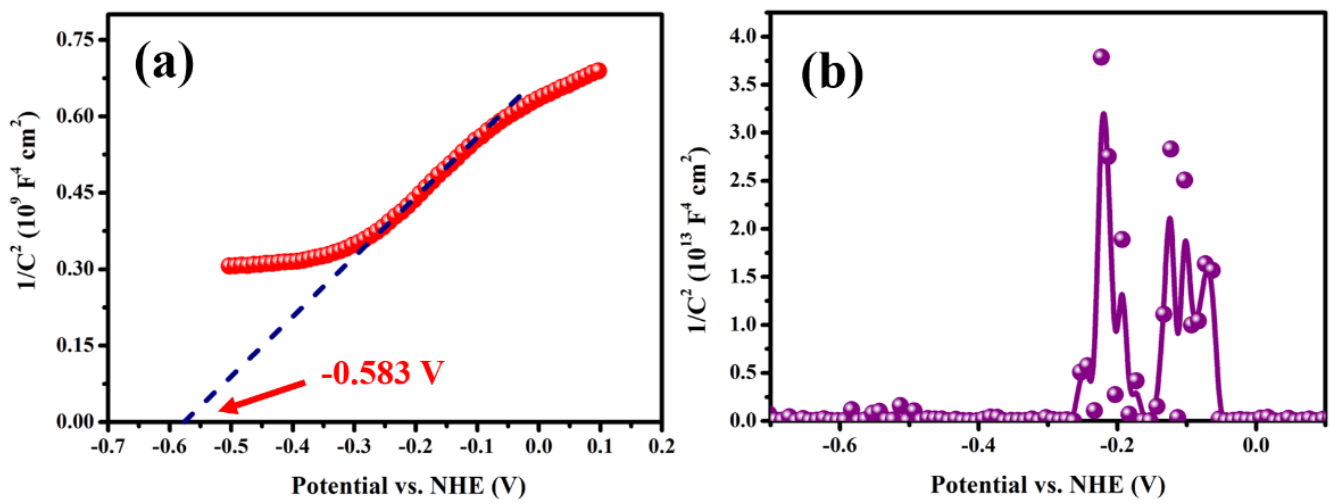

Figure S9. Mott-Schottky (MS) plots for (a) CdS and (b) CBP 6h measured in $0.5 \mathrm{M}$ $\mathrm{Na}_{2} \mathrm{SO}_{4}$ (aq) electrolyte. 


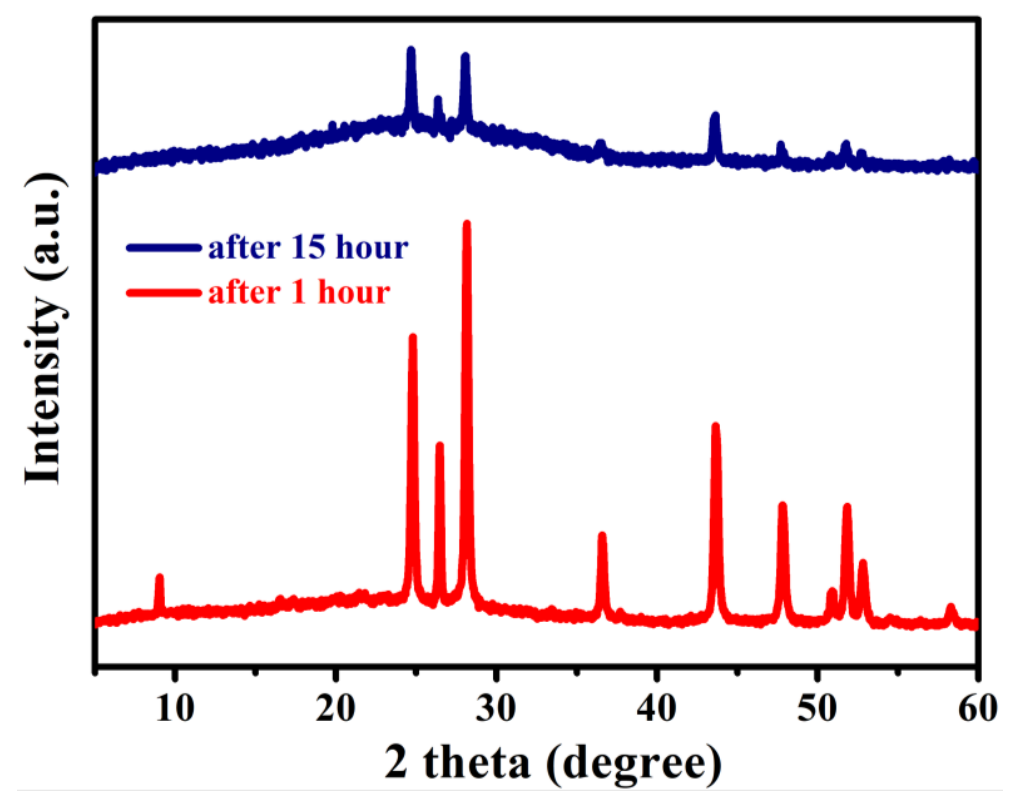

Figure S10. Comparison of XRD patterns for the CdS-CBP 6h sample after 1hour and 15-hour photocatalysis

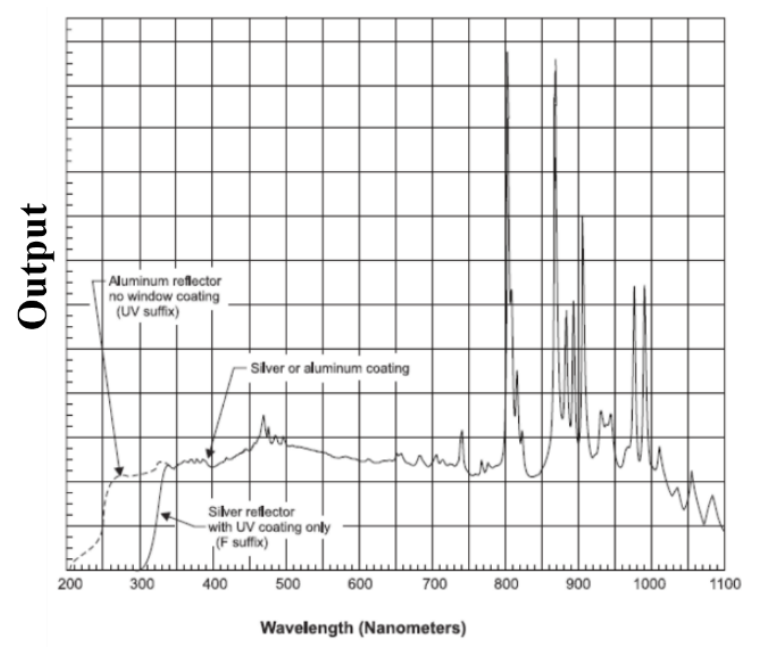

Figure S11. The illuminated spectrum of incident light for the lamp used in this work, provided by the factory. 


\section{References}

1. T. Hu, P. Li, J. Zhang, C. Liang and K. Dai, Applied Surface Science, 2018, 442, 20-29.

2. W. Jiang, X. Zong, L. An, S. Hua, X. Miao, S. Luan, Y. Wen, F. F. Tao and Z. Sun, ACS Catalysis, 2018, 8, 2209-2217.

3. J. Lv, J. Zhang, J. Liu, Z. Li, K. Dai and C. Liang, ACS Sustainable Chemistry \& Engineering, 2018, 6, 696-706.

4. X. Ding, Y. Li, J. Zhao, Y. Zhu, Y. Li, W. Deng and C. Wang, APL Materials, 2015, 3, 104410.

5. A. Meng, B. Zhu, B. Zhong, L. Zhang and B. Cheng, Applied Surface Science, 2017, 422, 518-527.

6. Y. Peng, G. Han, D. Wang, K. Wang, Z. Guo, J. Yang and W. Yuan, International Journal of Hydrogen Energy, 2017, 42, 14409-14417.

7. J. Ran, G. Gao, F.-T. Li, T.-Y. Ma, A. Du and S.-Z. Qiao, Nature Communications, 2017, 8, 13907.

8. J. Ran, B. Zhu and S. Z. Qiao, Angewandte Chemie International Edition, 2017, 56, 1037310377.

9. L. Zou, H. Wang and X. Wang, ACS Sustainable Chemistry \& Engineering, 2017, 5, 303-309.

10. S. Ma, J. Xie, J. Wen, K. He, X. Li, W. Liu and X. Zhang, Applied Surface Science, 2017, 391, $580-591$

11. F. Q. Zhou, J. C. Fan, Q. J. Xu and Y. L. Min, Applied Catalysis B: Environmental, 2017, 201, 77-83.

12. T. Di, B. Zhu, J. Zhang, B. Cheng and J. Yu, Applied Surface Science, 2016, 389, 775-782.

13. Z. Shi, X. Dong and H. Dang, International Journal of Hydrogen Energy, 2016, 41, 59085915.

14. L. J. Zhang, S. Li, B. K. Liu, D. J. Wang and T. F. Xie, ACS Catalysis, 2014, 4, 3724-3729. 